CLINICAL PRACTICE

\title{
Recommendations for the management of sickle cell disease in South Africa
}

\author{
N A Alli, M Patel, H D Alli, F Bassa, M J Coetzee, A Davidson, M R Essop, A Lakha, V J Louw, N Novitzky, V Philip, \\ J E Poole, R D Wainwright
}

Dr Nazeer Alli is principal consultant in the Department of Molecular Medicine and Haematology, National Health Laboratory Service (NHLS) and School of Pathology, University of the Witwatersrand, Johannesburg, South Africa; Prof. Moosa Patel is head of the Clinical Haematology Unit, Chris Hani Baragwanath Hospital, Johannesburg, and academic head of clinical haematology, Department of Medicine, Faculty of Health Sciences, University of the Witwatersrand; Dr Hassan Dawood Alli is a senior consultant in the Department of Opthalmology, St John's Eye Hospital, Johannesburg; Dr Fatima Bassa is a principal consultant and head of the Division of Clinical Haematology, Department of Medicine, Tygerberg Academic Hospital, Cape Town, South Africa; Associate Prof. Marius Coetzee is head of the Department of Haematology and Cell Biology, NHLS Universitas Tertiary Laboratories, University of the Free State, Bloemfontein, South Africa; Prof. Alan Davidson is head of Paediatric HaematologyOncology, Red Cross War Memorial Children's Hospital, Cape Town, and University of Cape Town; Prof. Mohammed Essop is head of the Department of Cardiology, Chris Hani Baragwanath Hospital, Johannesburg; Dr Atul Lakha is a consultant in the Department of Haematology, Chris Hani Baragwanath Hospital; Prof. Vernon Louw is head of the Department of Internal Medicine, University of the Free State; Prof. Nicolas Novitzky is head of the Division of Haematology, Department of Medicine and Pathology, Faculty of Health Sciences, University of Cape Town; Dr Vinitha Philip is a senior consultant in the Department of Haematology, Chris Hani Baragwanath Hospital; Prof. Janet Poole is head of the Department of Paediatric Haematology-Oncology, Charlotte Maxeke Johannesburg Academic Hospital, Johannesburg; and Dr Rosalind Wainwright is a consultant in and head of the Division of Paediatric Haematology-Oncology, Department of Paediatrics, Chris Hani Baragwanath Hospital.

Corresponding author: N A Alli (nazeer.alli@nhls.ac.za)

The spectrum of sickle cell disease (SCD) encompasses a heterogeneous group of disorders that include: (i) homozygous SCD (HbSS), also referred to as sickle cell anaemia; (ii) heterozygous SCD (HbAS), also referred to as sickle cell trait; and (iii) compound heterozygous states such as HbSC disease, HbS $\beta$ thalassaemia, etc. Homozygous or compound heterozygous SCD patients manifest with clinical disease of varying severity that is influenced by biological and environmental factors, whereas subjects with sickle cell trait are largely asymptomatic. SCD is characterised by vaso-occlusive episodes that result in tissue ischaemia and pain in the affected region. Repeated infarctive episodes cause organ damage and may eventually lead to organ failure. For effective management, regular follow-up with support from a multidisciplinary healthcare team is necessary. The chronic nature of the disease, the steady increase in patient numbers, and relapsing acute episodes have cost implications that are likely to impact on provincial and national health budgets. Limited resources mandate local management protocols for the purposes of consistency and standardisation, which could also facilitate sharing of resources between centres for maximal utility. These recommendations have been developed for the South African setting, and it is intended to update them regularly to meet new demands and challenges.

S Afr Med J 2014;104(11):743-751. DOI:10.7196/SAMJ.8470

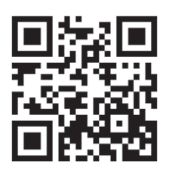

\section{Introduction}

Sickle cell disease (SCD) is the commonest inherited haemoglobin disorder, with approximately 70 million affected individuals worldwide. The highest gene frequencies are encountered in tropical regions, particularly equatorial Africa, the Arabian peninsula, India, the Mediterranean, the Caribbean and South America. The protective effect of the heterozygous state against malaria has led to persistence of the sickle gene in malarial endemic areas.

South Africa (SA) is not a malarial endemic area, and so has a predictably low prevalence of SCD $(<1 \%)$. However, with relaxed border controls since 1994, the influx of people from other African states has been accompanied by an influx of the sickle gene. Clinicians are therefore encountering an increasing number of patients with the disease.

Under conditions of decreased oxygen tension, HbS polymerises linearly in an alpha-helical fashion to form 'tactoids' that force red cells to elongate at two opposite ends and eventually assume a sickle shape (or variants thereof). Upon re-entry to the pulmonary circulation and subsequent reoxygenation of $\mathrm{HbS}$, sickle cells revert to their original shape. Repeated cycles of sickling and unsickling cause membrane damage and increased rigidity, which ultimately leads to formation of irreversibly sickled cells. Normal biconcave red cells are pliable and can easily squeeze through the microcirculation, but owing to their elongated shape, sickle cells are not as flexible. In addition, sickle cells have exposed receptors that bind to integrins on the endothelial surface, which makes them 'sticky'. In the presence of predisposing factors, e.g. infection, sickle cells therefore get trapped in the microcirculation and cause obstruction to flow, with resulting ischaemia. The severe pain that may be experienced at the site of ischaemia, commonly referred to as an 'acute pain crisis' or 'acute sickle crisis', is a hallmark of the disease. Chronic organ damage results from repeated infarctive episodes.

The only intervention that may be curative is stem cell transplantation (SCT), but this comes at considerable cost and requires expertise (Appendix 4). Gene therapy is experimental and if successful would overcome some of the hurdles of SCT. 


\section{Acute crises}

2.1 Acute vaso-occlusive crisis (VOC) (synonyms: acute pain crisis, acute sickle crisis ${ }^{[1]}$

Acute VOC is the hallmark of SCD, and is the most common acute presentation. Long bones are the most common sites for acute VOC, but any other site or organ may be involved, including the axial skeleton, abdomen, chest, kidneys, central nervous system and soft tissues.

In infants and young children, acute VOC involving small bones of the hands and feet (dactylitis), typically occurring between the ages of 6 months and 3 years, may be the first presenting manifestation of SCD. This causes acute pain as well as swelling and tenderness of the affected regions, often dubbed the 'hand-foot syndrome'.

Any drop in oxygen tension or related events can precipitate a sickle crisis. Precipitating factors include:

A. Acidosis, anaesthesia, anxiety, (high) altitude

B. Bouts of infection, bad habits, e.g. smoking, alcohol

C. Cold exposure

D. Dehydration

E. Exercise (vigorous)

F. Folate deficiency (e.g. megaloblastic crisis)

G. General surgery

H. Hypoxia

I. Infection

O. Other - trauma, menstruation.

Fever frequently accompanies a pain crisis. As the temperature rarely exceeds $38^{\circ} \mathrm{C}$, temperatures above this level should be investigated, guided by the clinical findings and further investigations, e.g. chest radiograph, and blood/urine culture. Empiric antibiotics should be administered until culture results are known, and adjusted where necessary.

Acute VOC often causes excruciating pain and can lead to despair and panic. Pain is experienced as a result of tissue ischaemia that results from sludging or occlusion of the microcirculation by sickled red cells. Vaso-occlusion of cerebral vessels results in clinical stroke or silent cerebral infarcts.

- Vigorous hydration with intravenous fluids (normal saline or 5\% dextrose in saline) is necessary.

- Check oxygen saturation and administer oxygen if $<95 \%$.

\subsubsection{Acute pain}

Pain management should be individualised, as patients have varying thresholds of pain and different levels of tolerance from past exposure to analgesics.

\section{Management:}

- Prehospital care: bed rest, hydration and simple oral analgesia should be instituted by the patient. Failure of self-treatment should mandate prompt referral to hospital (emergency department/ casualty/admission ward).

- Upon admission, rapid clinical assessment and reassurance are important.

- Analgesics should be administered as soon as possible, and within 30 minutes of arrival at hospital.

- For mild to moderate pain, paracetamol with codeine, nonsteroidal anti-inflammatory drugs (NSAIDs) or tramadol (tilidine in the case of children) may be used as single agents or in combination, bearing in mind the World Health Organization (WHO) three-step ladder for pain management:

- Step 1: Mild pain - non-opioid \pm adjuvant

- Step 2: Moderate pain - weak opioid (or low dose of strong opioid) \pm non-opioid \pm adjuvant

- Step 3: Severe pain - strong opioid \pm non-opioid \pm adjuvant)
- Opioids are generally required for severe pain. Morphine is the drug of choice, except where contraindicated (e.g. allergy, intracranial disease, liver disease, etc.). Ideally morphine should be administered parenterally at fixed intervals. Table 1 serves as a general guide to dosing.

Close monitoring of vital signs, pain control and sedation is essential at 15 - 20-minute intervals for the first hour, then at 30-minute intervals, and thereafter 2-hourly once pain control has been achieved. After $24-48$ hours of pain relief, parenteral morphine should be weaned off over the next 2 - 3 days. A nonopioid analgesic, such as paracetamol or an NSAID, may be added as an adjunct. For breakthrough pain, oral morphine solution can be used (e.g. 5 - $10 \mathrm{~mL} 4$ - 6-hourly). The frequency of and response to pain-relieving medication should be documented with pain charts or scales appropriate to the patient's age and cognitive abilities. Patient-controlled analgesia pumps are particularly useful for frequent, ongoing pain and should be offered where available. For morphine-related side-effects and their management, see Table 1.

Routine use of sedatives should be avoided. For extremely anxious and agitated patients, anxiolytics such as haloperidol, benzodiazepines or antihistamines may be used with caution.

Chronic pain is discussed under complications and organ damage (3.8).

\subsubsection{Stroke [2,3] $^{[2}$}

The incidence of stroke is highest between 2 and 11 years of age, with up to $30 \%$ of individuals being affected (the SA experience however, seems to suggest a lower incidence).

- Haemorrhagic stroke. Adults with SCD and moya-moya are at risk of recurrent intracranial haemorrhage. Bleeding aneurysms have been successfully treated with interventional neuroradiology (embolisation with coils) or surgery (craniotomy and clipping).

- Arterial stroke. In the acute phase, SCD patients with arterial stroke should undergo exchange transfusion, with the aim of achieving an $\mathrm{HbS}$ percentage $<30 \%$. As there is a high prevalence of a second stroke, it is recommended that these patients be put onto a chronic transfusion regimen, either top up or exchange transfusion (see below). There is no clear guidance on when to stop the transfusions.

- Primary stroke prevention. Long-term red cell transfusion is the mainstay of stroke prevention in SCD. It is possible to identify patients at high risk for developing stroke. Cerebral vessel velocities, as measured by transcranial Doppler (TCD) imaging, exceeding $200 \mathrm{~cm} / \mathrm{s}$ increase the risk (high risk) for developing stroke. The predictive value of TCD velocity is limited to children, as the velocity rarely exceeds $200 \mathrm{~cm} / \mathrm{s}$ in adults.

- Secondary stroke prevention. Following a first ischaemic stroke, the risk of recurrence in untreated cases is $50-92 \%$. This risk is reduced to about $10 \%$ by regular transfusions to maintain an HbS level $<30 \%$. The initial transfusion should be an exchange transfusion and may be followed by simple transfusions. Chronic transfusion is superior to hydroxyurea in stroke prevention.

- Covert infarction. The incidence of silent cerebral infarcts is 7.06/100 patient-years. There should be a high index of suspicion when patients present with cognitive abnormalities. Detection of cerebral infarcts is possible on magnetic resonance imaging (MRI).

- Epilepsy. There is a ten-fold increase in the occurrence of seizures in SCD compared with the general population. These are associated with silent infarcts and overt cerebrovascular disease. Seizures should be managed with anticonvulsants. 
Table 1. Suggested dosing of morphine for pain in SCD and management of side-effects

\begin{tabular}{|c|c|c|c|}
\hline & Morphine & Adult & Paediatric \\
\hline \multirow[t]{3}{*}{ Suggested dosing } & $\begin{array}{l}\text { Initial dose (administered until } \\
\text { pain control achieved) }\end{array}$ & $\begin{array}{l}0.1 \mathrm{mg} / \mathrm{kg} \text { IV every } 15-30 \mathrm{~min} \text { OR } \\
10 \mu \mathrm{g} / \mathrm{kg} / \mathrm{h} \text { as a continuous IV } \\
\text { infusion }\end{array}$ & $\begin{array}{l}0.1-0.15 \mathrm{mg} / \mathrm{kg} \text { ( } \max 7.5 \mathrm{mg} / \mathrm{dose}) \\
\text { Repeat morphine once after } 60 \mathrm{~min} \text { if } \\
\text { inadequate pain relief }\end{array}$ \\
\hline & $\begin{array}{l}\text { Subsequent dosing after pain } \\
\text { control achieved }\end{array}$ & $0.05-0.1 \mathrm{mg} / \mathrm{kg}$ IV every $2-4$ hours & $\begin{array}{l}\text { Additional IV boluses of morphine can } \\
\text { be given }-0.05 \mathrm{mg} / \mathrm{kg} 1 \text { - 2-hourly as } \\
\text { required }\end{array}$ \\
\hline & $\begin{array}{l}\text { If unable to obtain venous } \\
\text { access }\end{array}$ & $\begin{array}{l}\text { Initial dose: } 0.1 \mathrm{mg} / \mathrm{kg} \mathrm{SC} \text { followed by } \\
0.05-0.1 \mathrm{mg} / \mathrm{kg} \mathrm{SC} 2-4 \text {-hourly }\end{array}$ & $0.1-0.2 \mathrm{mg} / \mathrm{kg} \mathrm{SC} 4$-hourly \\
\hline \multirow[t]{4}{*}{ Side-effects } & Constipation & $\begin{array}{l}\text { Lactulose } 10 \mathrm{~mL} \text { bid OR } \\
\text { Senna } 2 \text { - } 4 \text { tablets daily }\end{array}$ & $\begin{array}{l}\text { Lactulose } 5 \mathrm{~mL} \text { bid } \\
\text { Senna } 1 \text { - } 2 \text { tabs daily ( }>6 \mathrm{yr})\end{array}$ \\
\hline & Nausea and vomiting & $\begin{array}{l}\text { Prochlorperazine } 5 \text { - } 10 \mathrm{mg} \text { tds OR } \\
\text { Cycline } 50 \mathrm{mg} \text { tds }\end{array}$ & $\begin{array}{l}\text { Prochlorperazine } 7.5 \mathrm{mg} \text { daily oral/ } \\
\text { rectal }\end{array}$ \\
\hline & Pruritus & $\begin{array}{l}\text { Hydroxyzine } 25 \mathrm{mg} \text { bid OR } \\
\text { Cetrizine } 10 \mathrm{mg} \text { nocte OR } \\
\text { Promethazine } 25 \mathrm{mg} \text { tds }\end{array}$ & $\begin{array}{l}\text { Hydroxyzine } 5 \text { - } 15 \mathrm{mg} / \text { day }(<6 \mathrm{yr}) \\
\text { Cetrizine } 5 \mathrm{mg} / \mathrm{d} \\
\text { Promethazine } 5 \text { - } 15 \mathrm{mg} / \text { day }(\mathrm{CI}<2 \mathrm{yr})\end{array}$ \\
\hline & Respiratory depression & $\begin{array}{l}\text { Stop opioid analgesics } \\
\text { Naloxone } 0.4-2 \mathrm{mg} \text { IV } \\
\text { Repeat when necessary }\end{array}$ & $\begin{array}{l}\text { Stop opioid analgesic } \\
\text { Naloxone } 0.01 \mathrm{mg} / \mathrm{kg} \mathrm{IV} \\
\text { Repeat with } 0.1 \mathrm{mg} / \mathrm{kg} \text { when necessary }\end{array}$ \\
\hline
\end{tabular}

2.1.3 Acute chest syndrome (ACS) ${ }^{[4]}$

ACS is the leading cause of death in SCD, and is characterised by:

- Fever - temperature $>38.5^{\circ} \mathrm{C}$

- Respiratory symptoms and signs, including cough, chest pain, dyspnoea or wheezing

- A new pulmonary infiltrate on chest X-ray (e.g. consolidation, segmental changes, etc.)

- ACS may begin as a pain crisis affecting the ribs, chest or shoulders. The pathogenesis involves vaso-occlusion, fat embolism and pulmonary thromboembolism. There is also a bronchoreactive component. Complications of an ACS include respiratory failure, central nervous system injury (from anoxia, infarction or haemorrhage), seizures, non-cardiogenic pulmonary oedema and multiorgan failure (from hypoxia, resulting in widespread sickling and VOC). Patients with ACS are best cared for in a high-care facility.

\section{Management of ACS:}

- Bed rest, hydration and analgesia

- Oxygen supplementation - oxygen may be administered to moderately hypoxaemic patients (arterial oxygen tension $70-80$ mmHg; oxygen saturation $92-95 \%$ ) nasally at a rate of $2 \mathrm{~L} / \mathrm{min}$

- Antibiotics are indicated as infection is a common precipitant of ACS, and should include a macrolide (e.g. erythromycin) to cover atypical organisms, combined with a second- or third-generation cephalosporin. The choice of antibiotics may be modified based on the age of the patient and the institutional sensitivity and resistance patterns.

- Bronchodilators should be prescribed for patients with evidence of bronchospasm.

- Blood transfusion. Where there is severe anaemia or significant drop in haemoglobin concentration ( $\geq 2 \mathrm{~g} / \mathrm{dL}$ ), a blood transfusion is required. An exchange transfusion is indicated if there is evidence of hypoxia $( \pm 5-10 \%$ drop from the baseline oxygen saturation level or $\mathrm{paO}_{2}<70 \mathrm{mmHg}$ ) and in deteriorating patients, since acute respiratory failure can supervene rapidly. Exchange transfusion in this setting often results in dramatic improvement.

- For severe hypoxia $\left(\mathrm{paO}_{2}<60 \mathrm{mmHg}\right.$ ), continuous positive airways pressure (CPAP) and mechanical ventilation may be necessary.

- Incentive spirometry is useful in preventing complications such as atelectasis and pulmonary infiltrates in patients experiencing pain above the diaphragm.

\subsubsection{Abdominal crisis}

An abdominal crisis most commonly arises from occlusion or infarction of mesenteric vessels and can mimic an acute abdomen, e.g. intestinal obstruction, cholecystitis, acute appendicitis or pancreatitis. Bowel ischaemia often causes abdominal pain that has a girdle distribution, called the 'girdle syndrome'. Ischaemic colitis causes thickening of the intestinal wall that can be detected on an abdominal computed tomography scan. Angiography does not image smaller vessels reliably and has limited diagnostic value.

Conservative management with rehydration and analgesia generally leads to resolution within $2-4$ days. Colonoscopy is indicated if infarction is suspected. Progression to severe ischaemic colitis necessitating colonic resection is rare and can be avoided by optimising colonic perfusion with exchange blood transfusion, intravenous broad-spectrum antibiotics and several days of bowel rest. However, there is a risk of bowel perforation if there is no response within 2 weeks. Patients who develop peritonitis, sepsis or gangrene (identified through endoscopy) or whose clinical condition deteriorates despite conservative management should undergo laparotomy for resection of the affected bowel segment.

\subsubsection{Priapism ${ }^{[5,6]}$}

Priapism is an unwanted, persistent, painful erection of the penis. By the age of 20 years, $30-45 \%$ of men with SCD will have suffered at least one episode of priapism. It can occur as an acute sustained attack or as recurrent shorter episodes (stuttering). If an episode lasts more than 3 - 4 hours, it can lead to erectile dysfunction. It is 
important for patients to be educated to abort attacks of priapism. Newer management ensures that more than $70 \%$ of men with SCD remain potent.

\section{Management principles of priapism include:}

- Simple measures: urination, hydration, hot baths, adequate analgesia (including morphine for severe pain in hospitalised patients - see management of acute pain above). Do not apply ice, as this will slow the circulation.

- Aspiration and irrigation of the corpora cavernosa.

- Intracavernous injection of sympathomimetic agents, such as phenylephrine (add $1 \mathrm{~mL}$ of a $10 \mathrm{mg} / \mathrm{mL}$ solution to $500 \mathrm{~mL}$ of $0.9 \%$ saline; administer $1 \mathrm{~mL}$ of the solution every 3 - 5 minutes for up to an hour, before proceeding to the next step), or as a second option epinephrine (add $1 \mathrm{~mL}$ of a 1:1 000 solution to $1000 \mathrm{~mL}$ saline).

- Surgical shunting (performed by a urologist) would be indicated for ongoing, refractory ischaemic priapism.

- Once the acute episode has settled, review and optimise the comprehensive care of the patient.

- If stuttering priapism persists despite optimal treatment, specific prophylaxis and home treatment should be considered. This includes oral pseudoephedrine ( $30 \mathrm{mg}$ at night if $<10$ years of age $60 \mathrm{mg}$ at night if $>10$ years) or oral etilefrine $(25 \mathrm{mg}$ at night for adults).

- A urologist and a clinical psychologist should be involved in the management of patients who develop erectile dysfunction. There are various medical and prosthetic options available for such patients.

\subsection{Splenic sequestration crisis}

This is a life-threatening emergency where the spleen enlarges very rapidly, sometimes within hours, and causes massive pooling of blood. The effect is synonymous with internal exsanguination with development of acute anaemia.

Splenic sequestration is most common in early childhood but may be encountered in older children and adults, particularly those with compound heterozygous sickle- $\beta$ thalassaemia or HbSC disease.

Early and urgent blood transfusion is life-saving. Splenectomy is generally indicated for recurrent life-threatening episodes (usually after 4 - 6 years of age).

When the spleen regresses to its original size, the blood returns to the main circulation, which may increase the haemoglobin to above baseline levels. This is known as reverse sequestration and poses a risk for developing acute cardiac failure, acute hypertension or stroke. Care should therefore be taken not to aim for baseline haemoglobin levels during transfusion.

Education of parents to palpate for splenomegaly from infancy/ early childhood allows for early recognition and prompt intervention of this emergency situation. ${ }^{[7]}$

\subsection{Aplastic crisis}

In the context of SCD, as in other haemolytic states, aplastic crisis refers to pure red cell aplasia, where there is selective erythroid hypoplasia or aplasia with resultant reticulocytopenia. The most common cause is infection with parvovirus type B19, which infects early erythroid precursors through the glycophorin receptor. In normal individuals this infection rarely causes anaemia, but in haemolytic states where there is maximal erythropoietic activity, destruction of erythroid precursors leads to rapid development of severe anaemia. Blood transfusion is the mainstay of management. Intravenous gamma globulin and/or corticosteroids may be indicated for the treatment of parvovirus B19 infection.

\subsection{Haemolytic crisis}

Haemolytic crisis may be caused by an acute VOC, malarial infection or oxidant drug exposure in individuals with concomitant glucose-6-phosphate dehydrogenase (G6PD) deficiency. Haemolytic crisis may be distinguished from aplastic crisis by the finding of a reticulocytosis as opposed to a reticulocytopenia. Rarely, a hyperhaemolysis syndrome may be triggered by a red cell transfusion. Here the haemoglobin concentration drops to below pretransfusion levels as a result of destruction of allogeneic as well as autologous red cells without any evidence of an antibodymediated transfusion reaction. The mechanism of hyperhaemolysis is not known, but it is thought to result from hyperactive macrophage activity. Corticosteroids (first line) and intravenous immunoglobulin are the mainstays of treatment. Since red cell transfusion during an episode of hyperhaemolysis can accelerate the haemolytic process, additional transfusions should be limited to situations of life-threatening anaemia.

\section{Complications and organ damage 3.1 Infection} Increased susceptibility to infection in SCD patients may be due to a number of predisposing factors, including functional asplenia (hyposplenism), impaired complement activity, zinc deficiency, iron overload and the presence of necrotic tissue. The spleen is an early casualty of organ damage from repeated infarctive episodes, which reduce it to a nubbin of fibrous tissue. Functional asplenia in particular increases the propensity to infections with encapsulated bacteria and malaria, which may be life-threatening. Key to a successful outcome is early recognition and prompt therapeutic intervention.

Osteomyelitis is often a result of vaso-occlusion, ischaemia and infarction, with consequent bone necrosis and secondary infection. In addition, bacteraemia may lead to osteomyelitis or septic arthritis.

\section{Management of osteomyelitis entails:}

- Supportive care including bed rest, splinting of the affected limb, analgesia, hydration and oxygenation

- Surgical drainage where necessary

- Administration of intravenous antibiotics for 2 - 6 weeks. Appropriate antibiotics include the cephalosporins such as ceftriaxone and the quinolones in Salmonella osteomyelitis, and cloxacillin where Staphylococcus aureus is suspected. See below for preventive measures against infection.

\subsection{Cardiovascular complications}

Cardiovascular complications have become more evident with increasing longevity in patients with SCD and include pulmonary hypertension, left and right ventricular dysfunction, myocardial infarction, arrhythmias and sudden death. ${ }^{[8]}$

\subsubsection{Pulmonary hypertension (PHT) ${ }^{[9]}$}

PHT occurs in $5-30 \%$ of patients with SCD and is a risk factor for sudden cardiac death. Although the precise mechanism has not been defined, chronic haemolytic anaemia and release of free haemoglobin, which inactivates nitric oxide, may have an important role in the causation of pulmonary vasculopathy. Proliferation of smooth-muscle cells with resultant vascular obliteration leads to a pathological picture almost identical to that seen in idiopathic PHT. Other factors that contribute to the development of PHT in this population include functional asplenia, thromboembolism, lung fibrosis, hypoxia and left ventricular dysfunction. 
Echocardiography is the most useful screening test when PHT is suspected on clinical grounds, but it should be emphasised that definitive confirmation requires invasive right-heart catheterisation. The echocardiographic definition of PHT is a pulmonary artery systolic pressure $>40 \mathrm{mmHg}$, while the haemodynamic definition of PHT is a mean pulmonary artery pressure (MPAP) $>25 \mathrm{mmHg}$ at rest, measured at cardiac catheterisation. A raised tricuspid regurgitant velocity (TRV) $(>2.5 \mathrm{~m} / \mathrm{s})$ on Doppler echocardiography correlates well with an increased MPAP, and is more suitable for regular screening of patients owing to the non-invasive nature of the test. However, a diagnosis of PHT based on an elevated TRV needs to be confirmed with cardiac catheterisation, as not all patients with a raised TRV have PHT. Several studies have confirmed that a TRV $>2.5 \mathrm{~m} / \mathrm{s}$ is associated with a $9-16$ times increased risk of death. These patients should be referred to a cardiologist for further management and follow-up.

\section{Therapeutic interventions include:}

- Prostacyclin analogues (e.g. epoprostenol, iloprost)

- Phosphodiesterase type 5 inhibitors (e.g. sildenafil)

- Endothelin receptor antagonists (e.g. bosentan).

\subsubsection{Left ventricular dysfunction (LVD)}

LVD is not infrequent and is largely related to the duration and severity of anaemia. Compensatory left ventricular hypertrophy and dilatation are accompanied by both systolic and diastolic dysfunction and consequent heart failure. Other factors contributing to the heart failure phenotype include systemic hypertension, chronic inflammation, and possibly direct myocardial damage as a result of microvasculopathy.

The treatment of LVD and heart failure should follow standard guidelines and include diuretics, digitalis, beta-blockers and angiotensin-converting enzyme (ACE) inhibitors.

\subsubsection{Myocardial infarction (MI)}

MI has been documented in SCD, but the mechanisms are unclear because coronary angiography rarely reveals obstructive coronary disease. Other mechanisms that have been postulated include microvascular obstruction and endothelial dysfunction. The role of standard therapies for MI such as antiplatelets and thrombolytics remain unclear, although most patients improve with standard management of the sickle cell crisis.

\subsection{Renal complications}

Patients may present with symptoms early on in life and may manifest with the following complications:

\subsubsection{Hyposthenuria}

Hyposthenuria refers to an inability to concentrate urine maximally and is a common renal abnormality in SCD. Hyposthenuria typically manifests as enuresis in early childhood (in rare cases it may continue into the teenage years) and may cause nocturia in adults. Maintaining good hydration is important, as these patients are more susceptible to dehydration than normal individuals.

\subsubsection{Haematuria}

Haematuria may occur in homozygous SCD (HbSS) as well as heterozygous SCD (HbAS) and is caused by renal papillary necrosis resulting from ischaemic damage to the renal medulla. Treatment involves bed rest, maintenance of a high urinary flow, and blood transfusion if blood loss is significant. In the event of excessive or life-threatening bleeds, local resection of the bleeding segment in the kidney is preferable. It is, however, important to exclude other causes of haematuria.

\subsubsection{Proteinuria}

Screening for proteinuria should be performed annually with a urine dipstick. If positive, a 24-hour protein estimation or urine albumin/creatinine ratio is indicated. For patients with albuminuria, ACE inhibitors or angiotensin-II receptor antagonists are usually beneficial.

Renal function should be monitored annually (urine for microalbuminuria, blood urea, electrolyte and creatinine levels). Serum creatinine levels are generally low in SCD, so a rise in the creatinine level to within the normal range often signals the onset of renal impairment. Long-term use of NSAIDs should be avoided, particularly in patients with evidence of renal impairment. Renal transplantation may be considered in patients with end-stage renal failure.

Patients with renal dysfunction should be assessed and followed up by a renal physician.

\subsection{Avascular necrosis (AVN)}

Osteonecrosis or AVN occurs when repetitive vaso-occlusion results in the infarction of articular surfaces and juxta-articular bone, e.g. the head of a long bone. The most common sites are heads of femoral bones and humeri, followed by the knee (tibial condyles) and small joints of the hands and feet. The femoral head is highly vulnerable to ischaemic damage because its blood supply depends solely on an endartery. Multiple joints are often affected. Pain due to AVN is most noticeable during weight bearing and there may also be functional limitation of the joint. Early disease is best detected by MRI (signal intensity changes, irregular bony and articular outline). Plain X-rays detect only more advanced disease (flattening of the head, coarsening of the bone architecture, cystic areas, sclerosis). Isotope bone scans may detect osteonecrosis as focal areas of increased activity. Without treatment it is expected that most affected femoral heads will collapse within 5 years of diagnosis. Treatment options include a more conservative approach in children, with analgesia (paracetamol, mild opiates, NSAIDs), physiotherapy and protected weight bearing (avoidance of weight bearing) for approximately $3-6$ months. Secondary degenerative arthritis can compound the osteonecrosis in adults and a conservative approach is less effective. Analgesia, core decompression and realignment osteotomy with aggressive physiotherapy are used in early disease, while joint replacement is reserved for patients with severe manifestations of advanced disease. There is a high rate of perioperative complications, which include blood loss, acute chest syndrome, infection and failure of the prosthesis.

\subsection{Leg ulceration}

Leg ulceration is a frequent complication of SCD in adults and most commonly occurs over the shins and malleoli. Leg ulcers should be treated promptly to minimise progression to a chronic state.

Investigation and management of leg ulcers include the following: - Swab for culture if infected, and treat as per sensitivity results. Osteomyelitis should be excluded if there is fever and bone tenderness. An MRI scan may be indicated to establish a diagnosis of osteomyelitis.

- Topical emollients and wound dressings to promote moist wound healing. Graduated compression bandages will reduce venous hypertension. Zinc supplementation may be of benefit in terms of wound healing, as the patients tend to be in negative zinc balance. Topical antibiotics should be avoided to prevent sensitisation.

- Arterial insufficiency should be excluded. If discovered, the patient must be referred to a vascular surgeon. 
- For those who fail to respond to the above measures, the attending doctor should consider:

- Regular blood transfusions until wound healing occurs (a costly option)

- Wound dressings with Thrombostim, a product developed and manufactured by the South African National Blood Service (SANBS). Thrombostim is a dual-component treatment system containing lyophilised platelet-rich plasma, which is rich in platelet-derived growth factor (PDGF), and lyophilised thrombin. PDGF is essential for the wound healing process.

The surgical team should be involved in the management of patients with chronic leg ulcers.

\subsection{Gallstones}

Formation of gallstones is a common complication of SCD, and if the patient is symptomatic, cholecystectomy should be performed. A laparoscopic procedure is preferred.

\subsection{Ocular complications}

In the early stages ocular complications are usually clinically silent. Anterior- and posterior-segment changes of the eye can occur. Retinal changes are broadly categorised as follows:

\subsubsection{Non-proliferative retinopathy}

Non-proliferative retinopathy includes 'salmon patches' and 'black sunbursts. These changes occur as a result of peripheral retinal haemorrhages due to arteriolar occlusion and rupture of the blood vessel. This does not normally lead to visual loss.

\subsubsection{Proliferative retinopathy}

Proliferative retinopathy is predominantly encountered in young adults with SCD (including HbSS and HbSC disease). Neovascularisation occurs in response to microvascular occlusion and ischaemia. This may lead to complications such as vitreous haemorrhage and retinal detachment, which is a cause of sudden loss of vision.

Pan-retinal photocoagulation laser therapy is effective in preventing vision-threatening complications such as vitreous haemorrhage and retinal detachments. Antivascular endothelial growth factor (antiVEGF) agents such as bevacizumab have also been used in the treatment of proliferative retinopathy.

Annual ophthalmic assessment is recommended.

\subsection{Chronic pain}

A multidisciplinary team is required for the optimal management of chronic pain. The cause of the pain should be determined. Where circumstances permit, home management should be encouraged.

- Pharmacological management should include medium- to long-acting opioids, as the use of frequent short-acting opioids is less effective. NSAIDs should be used judiciously with 3-monthly monitoring of renal function. For breakthrough pain, alternative analgesia such as fentanyl or oxycodone may be used, where available.

- Non-pharmacological measures should be considered where necessary, such as physiotherapy, acupuncture, etc.

- Surgical options may be exercised where indicated, e.g. decompression or total hip replacement for avascular necrosis of the hip and cholecystectomy for gallstones. Perioperative thromboprophylaxis is imperative.

- Chronic headache is common in SCD, and any exacerbation or change in the nature of the pain warrants neurological assessment (to exclude potentially life-threatening events such as intracranial haemorrhage or venous sinus thrombosis).

\section{Preventive measures \\ 4.1 Patient education}

Patients as well as caring family members need to be informed about the disease process, with particular attention being paid to the following:

- Precipitating factors

- Home management. The aim is to recognise symptoms or signs that require hospital admission, and at the same time abort the various forms of acute crises. A supply of simple analgesics should be made available for managing milder forms of sickle crises. Parents should be taught the art of palpating for the spleen in order to pre-empt a splenic sequestration crisis.

- Genetic counselling.

\subsection{Infection control}

Globally, infection is the leading cause of death in developing countries. The factors responsible for increased susceptibility to infections in SCD have been mentioned previously. In particular, hyposplenism renders patients more susceptible to the detrimental effects of encapsulated organisms and malaria. In addition, infection is a common precipitant of an acute crisis, and prompt treatment with appropriate antibiotics is therefore essential.

\subsubsection{Antibiotics}

There is debate on the prophylactic use of oral antibiotics in all patients with SCD. However, penicillin VK $125 \mathrm{mg}$ twice daily orally for children under 3 years of age and $250 \mathrm{mg}$ bid for children older than 3 years of age is recommended, and continued until adolescence. Erythromycin is recommended for patients who are allergic to penicillin.

\subsubsection{Immunisation}

- Pneumococcal vaccine. Adults and children $>2$ years of age should receive the unconjugated vaccine (Pneumovax $23^{\circ}$ ). Conjugated vaccine (Prevenar $13^{\circ}$ ) is provided to children under the age of 2 years as part of the national immunisation schedule. Children who have missed the conjugated vaccine should have two doses of the conjugated vaccine followed by a dose of the unconjugated vaccine, at 6 - 8-week intervals.

- Conjugated meningococcal $\mathbf{C}$ vaccine. If not given as part of the childhood immunisation programme, a single dose should be given.

- Haemophilus influenzae type B vaccine. If not given as part of the childhood immunisation programme, a single dose should be given.

- Annual influenza vaccination is recommended.

\subsection{Nutritional supplements}

- Folic acid becomes depleted owing to its high turnover as well as loss from chronic haemolysis. A dose of 1 - $2 \mathrm{mg}$ daily is adequate to supplement the increased requirements of folate.

- Zinc is a constituent of the metalloenzyme carbonic anhydrase, which is lost in intravascular haemolytic states. Zinc should be supplemented in individuals displaying symptoms of zinc deficiency, viz. malaise, listlessness and fatigue or repeated infections.

- Repeated transfusions can lead to iron overload. However, individuals who are not regularly transfused can develop a negative iron balance, necessitating supplementation.

\subsection{Family screening}

Family members and prospective partners should be screened. Cultural and societal sensitivities often pose a challenge to effective screening programmes, largely owing to stigma associated with the 
disease. An aggressive education drive is necessary to overcome such barriers. The most convenient method of screening for SCD is high-performance liquid chromatography (HPLC), since it is reliable, automated and allows for high throughput. Hb electrophoresis may be used as a confirmatory test. The slide sickle and solubility tests, although still used in some under-resourced centres, are far from ideal because they may not distinguish HbAS from HbSS and also cannot detect compound heterozygous states.

\subsection{Antisickling agents}

There are various antisickling agents, such as hydroxyurea (HU), statins, clotrimazole and butyrate. Of the available agents, HU has proven to be the most effective. HU is a cytotoxic agent that acts by inhibiting the enzyme ribonucleotide reductase. The mechanisms of action of hydroxyurea include:

- Increase in $\mathrm{HbF}$ concentration. $\mathrm{HbF}$ does not participate in formation or elongation of the sickle tactoids and as such retards the sickling process.

- Increase in the mean cell volume, which prolongs the delay time (time taken for a red cell to sickle).

- Reduction of cell counts and adhesion molecules, thereby improving the rheology and flow of blood.

HU is indicated in all adult patients with SCD. Use in children has been less definitive, however, primarily owing to concerns regarding fertility and potential mutagenicity. There is no clear-cut evidence that long-term use of $\mathrm{HU}$ (when used for the treatment of benign disorders) leads to an increased risk of secondary malignancies. Although infertility is not a recognised complication, reduced sperm counts and a decrease in sperm motility have been demonstrated, which appear to improve or reverse after discontinuation of HU. On the contrary, numerous studies have demonstrated the benefits of $\mathrm{HU}$, viz. a decreased number of acute crises, decreased transfusion requirements and organ preservation in children. Institution of HU therapy is therefore recommended in children with $\mathrm{SCD}>2$ years of age. ${ }^{[7]}$ The dosage scheme of $\mathrm{HU}$ is as follows: Start at $10-15 \mathrm{mg} / \mathrm{kg} / \mathrm{d}$ and increase to a maximum of $30 \mathrm{mg} / \mathrm{kg} / \mathrm{d}$. The dose should be titrated against the neutrophil count and increased by $5 \mathrm{mg} / \mathrm{kg}$ increments at 3-monthly intervals. The dose should be decreased if the neutrophil count drops below $1.5 \times 10^{9} / \mathrm{L}$, and stopped if it drops below $1 \times 10^{9} / \mathrm{L}$ or when conception is planned.

\subsection{Gout prevention}

Elevation of uric acid levels are closely linked with the degree of haemolysis. There may also be a contribution from the use of hydroxyurea or renal dysfunction. A uric acid-lowering agent such as allopurinol is recommended where the uric acid levels are elevated.

\subsection{Outpatient visits}

Regular outpatient visits are essential for monitoring and early detection of organ dysfunction. (See Appendix 3 for a recommended schedule of outpatient visits.)

Disclaimer. The advice and information in these guidelines is largely evidence based and believed to be true and accurate at the time of going to press. The authors or publishers therefore cannot accept any legal responsibility for these recommendations. It should also be noted that, for reasons of conscience, not all the authors agree on termination of pregnancy beyond situations where the life of the pregnant woman is endangered.
References. Please note that only key references are cited as per publisher's requirements. Detailed referencing is available on request from the authors.

\footnotetext{
1. Rees DC, Olujohungbe AD, Parker NE, et al. Guidelines for the management of acute painful crisis in sickle cell disease. Br J Haematol 2003;120(5):744-752. [http://dx.doi.org/10.1046/j.1365141.2003.04193.x]

2. Adams RJ, McKie VC, Hsu L, et al. Prevention of a first stroke by transfusions in children with sickle cell anaemia and abnormal results on transcranial Doppler ultrasonography. N Engl J Med 1998;339(1):5-11. [http://dx.doi.org/10.1056/NEJM199807023390102]

3. Ware RE, Helms RW. SWITCH investigators. Stroke with transfusions changing to hydroxyure (SWITCH). Blood 2012;119(17):3925-3932. [http://dx.doi.org/10.1182/blood-2011-11-392340]

4. Vichinsky EP, Neumayr LD, Earles AN, et al. Causes and outcomes of the acute chest syndrome in sickle cell disease. National Acute Chest Syndrome Study Group. N Engl J Med 2000;342(25):18551865. [http://dx.doi.org/10.1056/NEJM200006223422502]

5. Emond AM, Holman R, Hayes RJ, et al. Priapism and impotence in homozygous sickle cell disease Arch Intern Med 1980;140(11):1434-1437. [http://dx.doi.org/10.1001/archinte.1980.00330220022011]

6. Montague DK, Jarow J, Broderick GA, et al. American Urological Association guideline on the management of priapism. J Urol 2003;170(4):1318-1324. [http://dx.doi.org/10.1097/01 ju. $0000087608.07371 . \mathrm{ca}$

7. Wang WC, Ware RE, Miller ST, et al., for the BABY HUG investigators. Hydroxycarbamide in very young children with sickle cell anaemia: A multicentre, randomised, controlled trial (BABY HUG). Lancet 2011;377(9778):1663-1672. [http://dx.doi.org/10.1016/S0140-6736(11)60355-3]

Gladwin MT, Sachdev V. Cardiovascular abnormalities in sickle cell disease. J Am Coll Cardio 2012;59(13):1123-1133. [http://dx.doi.org/10.1016/j.jacc.2011.10.900]

Gladwin MT, Sachev V, Jison ML, et al. Pulmonary hypertension as a risk factor for death in patients with sickle cell disease. N Engl J Med 2004;350(9):886-895. [http://dx.doi.org/10.1056/NEJMoa035477] Oteng-Ntim E, Babarinsa I, Bagot C, et al. Management of Sickle Cell Disease in Pregnancy. Green-top Guideline No. 61. London: Royal College of Obstetricians and Gynaecologists, 2011:1-20

1. Smith LA, Espeland M, Bellevue R, et al. Pregnancy in sickle cell disease: Experience of the Cooperative Study of Sickle Cell Disease. Obstet Gynecol 1996;87(2):199-204. [http://dx.doi.org/10.1016/00297844(95)00367-3]

12. Wenko SO, Telen MJ. Transfusion management in sickle cell disease. Hematol Oncol Clin North Am 2005;19(5):803-826. [http://dx.doi.org/10.1016/j.hoc.2005.07.002]

3. Bolton-Maggs PHB. Transition of care from paediatric to adult services in haematology. Arch Dis Child 2007;92(9):797-801. [http://dx.doi.org/10.1136/adc.2006.103804]

14. Lucarelli G, Clift RA, Galimberti M, et al. Bone marrow transplantation in patients with thalassemia. $\mathrm{N}$ Engl J Med 1990;322(7):417-421. [http://dx.doi.org/10.1056/NEJM199002153220701]

15. Panepinto JA, Walters MC, Carreras J, et al. Matched-related donor transplantation for sickle cell disease: Report from the Center for International Blood and Transplant Research. Br J Haematol 2007;137(5):479-485. [http://dx.doi.org/10.1111/j.1365-2141.2007.06592.x]
}

Accepted 20 June 2014

\section{Appendix 1. Pregnancy and contraception in SCD}

Pregnancy is a major decision for a woman with SCD, since it is associated with a number of maternal and fetal complications. Maternal complications include VOC (30 - 50\%), infections (28\%), anaemia (34\%), pre-eclamptic toxaemia (PET)/eclampsia (9\%), preterm labour (24\%), etc. Fetal complications include miscarriage, abruptio placentae, placenta praevia, preterm delivery (24\%), low birth weight (20\%), fetal distress (14\%), stillbirths (0.9\%), etc.

Improvements in obstetric and perinatal care have resulted in a decrease in maternal and perinatal mortality (from $11.5 \%$ in the 1970 s to $<2 \%$ ). The philosophy of avoidance of pregnancy in the past has now shifted to one of support and comprehensive stepwise care, for which a multidisciplinary team including the haematologist and obstetrician is required. This is summarised below.

\section{Management of SCD in pregnancy ${ }^{[10,11]}$}

1. Screening and counselling of young women at risk

- Education

- Issues surrounding fertility, contraception and pregnancy

- Choice and screening of spouse/partner

- Discussion of potential maternal and fetal complications.

- Planning of the pregnancy prior to conception - discuss discontinuation of $\mathrm{HU}$ prior to conception, pneumococcal vaccination, and need for continuation or termination of pregnancy (TOP).

2. Antenatal diagnosis. This is not without risk of complications and should be undertaken if TOP is a realistic option. Techniques include chorionic villous sampling at $9-13$ weeks, amniocentesis at $15-16$ weeks and fetal blood sampling at $18-20$ weeks. 
3. Antepartum management

- Early booking is advised, ideally by 12 - 14 weeks. Recommended followup frequency: monthly until 28 weeks, fortnightly until 34 weeks and weekly after 34 weeks.

- Close monitoring of mother and fetus is needed for early detection of recognised complications. Perform growth scans/ ultrasound monthly until 34 weeks, then fortnightly until delivery. Weekly nonstress testing from 32 weeks' gestation is recommended.

- Folic acid $5 \mathrm{mg}$ daily, and iron supplementation if indicated.

- Blood transfusion - prophylactic transfusion for uncomplicated pregnancy is not recommended. Indications for blood transfusion include: anaemia associated with cardiorespiratory disease, acute chest syndrome, refractory PET, preparation for caesarean section (CS) and other medical or obstetric indications.

\section{Labour and delivery}

- Timing and route of delivery should be based on obstetric indications as in nonSCD cases.

- During labour ensure good pain relief, adequate hydration, oxygen, and fetal and maternal monitoring.

- Avoid long and exhausting labour and delivery.

- For CS, optimise surgical and anaesthetic risks (e.g. transfusion, hydration, oxygenation, temperature control, etc.). Beware of acute chest syndrome post-surgery (peak 48 hours post surgery).

- Where opiates are used, be aware of the sedative and depressive effects. If they are used prior to or during labour and delivery, a neonatologist should be alerted, and be present at delivery in case of fetal sedation.

\section{After delivery}

- Owing to the increased risk of thromboembolic disease, aim for early ambulation and use of stockings, with thromboprophylaxis in high-risk individuals.

- During breastfeeding, avoid HU and ensure good hydration.

- It is important to offer family planning advice with the various options on contraception, viz. oral contraceptive pill (including combined and progesteroneonly pill), depot medroxyprogesterone acetate, barrier methods and copper intrauterine contraceptive devices, which are all acceptable in SCD.
6. Neonate

- Establish early diagnosis - an umbilical cord blood sample may be used for convenience.

- In the case of a child born with SCD, institute a comprehensive care programme where both the paediatrician and the haematologist are involved in management.

\section{Appendix 2. Blood transfusion and iron overload in $\mathrm{SCD}^{[12]}$}

Blood transfusion remains an important component of the management of patients with SCD. The rationale behind transfusion is to improve microvascular perfusion and oxygen-carrying capacity and decrease the proportion of sickle cells in the circulation. Indications for transfusion are set out in Table 2.

Transfusion for anaemia is generally indicated in symptomatic patients with a $>20 \%$ drop in haemoglobin or a decrease in oxygen saturation. The goal of transfusion should be to reduce the level of $\mathrm{HbS}$ to $<30 \%$ and in order to prevent the problems of hyperviscosity, achieve a post-transfusion haemoglobin concentration not exceeding 9 - $10 \mathrm{~g} / \mathrm{dL}$.

The role of transfusion in stroke is discussed under 'Acute VOC' (2.1.2).

Controversial indications for transfusion include priapism, pregnancy and patients with chronic leg ulcers (cf. respective sections).

Simple v. exchange transfusion. The decision with regard to each of the modalities of transfusion will depend on the indication, clinical circumstances and available resources. Exchange transfusion has the added advantage of reducing $\mathrm{HbS}$ effectively without the risk of increasing viscosity or causing volume overload. Simple transfusion has the advantage of ease of administration, as well as accessibility outside major medical centres. The disadvantages include the potential to increase viscosity, as well as the risk of iron and volume overload.

The optimal management strategy should also include measures to limit the complications associated with blood transfusion, the most important being alloimmunisation and iron overload. Alloimmunisation is a commonly encountered complication in patients with SCD, and extended phenotyping has been shown to reduce the incidence of this complication. Testing for at least $\mathrm{D}$, $\mathrm{C} / \mathrm{c}, \mathrm{E} / \mathrm{e}$ and $\mathrm{K}$ antigens is recommended before commencing transfusions, and phenotypically matched, leucodepleted blood should be administered.

Patients receiving regular transfusions should have regular monitoring of their iron status, and chelation therapy should be commenced appropriately. The iron chelators available are deferoxamine (given parenterally), deferiprone and deferasirox (given orally). Oral iron chelators are preferred, as they improve patient compliance. Deferiprone should be avoided in patients with aplastic crisis or neutropenia because it is known to cause agranulocytosis in some patients.

\section{Appendix 3. Outpatient follow-up and transition of care in SCD \\ Outpatient follow-up}

In the initial stages, patients should be seen at the haematology outpatient clinic on a monthly basis. Intervals between visits may be increased to 3 months once the patient is stable and therapy is optimised.

Investigations conducted at outpatient level for monitoring purposes are listed in Table 3 .

\section{Transition of care ${ }^{[13]}$}

Transfer of care from a paediatric to an adult team is a major life-changing event for the young patient with $\mathrm{HbSS}$.

With advances in medical care, $>90 \%$ of patients with SCD now reach adulthood, with the majority living into their forties.

\section{Table 2. Indications for blood transfusion in SCD}

\section{Acute}

Symptomatic anaemia

Splenic/hepatic sequestration crisis

Acute chest syndrome

Acute neurological syndromes

Perioperative (major surgery)

Acute multiorgan failure

Sepsis

$\mathrm{SCD}=$ sickle cell disease

\section{Chronic}

Primary or secondary stroke prevention

Pulmonary hypertension

Recurrent sequestration crisis/chest syndrome Anaemia associated with chronic renal failure Congestive cardiac failure 
Table 3. Guide to outpatient monitoring of SCD

\begin{tabular}{|c|c|c|c|c|c|}
\hline & Baseline & Monthly & 6-monthly & Annual & Biennial \\
\hline $\mathrm{FBC}+$ differential & $\times$ & $x$ & & & \\
\hline $\mathrm{U}+\mathrm{E}$ & $x$ & & $x$ & & \\
\hline LFTs & $x$ & & & $\times$ & \\
\hline Urine for microalbumin & $x$ & & $x$ & & \\
\hline Chest X-ray & $x$ & & & & $x$ \\
\hline TRV $(>10 \mathrm{yr})$ & $x$ & & & & $x$ \\
\hline Lung function tests ( $>10 \mathrm{yr})$ & $x$ & & & & $x$ \\
\hline TCD (paediatric population) & $x$ & & & $x$ & \\
\hline Ocular assessment & $x$ & & & $\times$ Adults & $\begin{array}{l}\times \text { Older children with } \\
\text { HbSC }\end{array}$ \\
\hline Serum ferritin (if regularly transfused) & & & $x$ & & \\
\hline
\end{tabular}

Longer survival has increased the need for successful transition of care from paediatric to adult services.

The handover from paediatric to adult services should be planned and managed as a process and should begin after the age of 12 years. The optimal time for transition should be individualised and requires assessment of the level of readiness as well as patient and parent education about their illness and the transition process. The anxiety of patients during a transfer process centres around transfer of their history, the adult service's attitude to issues pertaining to pain management, and practical issues such as appointments, etc.

Methods to facilitate the process include printed literature, meeting with a support group or someone who has successfully transitioned, visiting the adult service before the first appointment, and the establishment of special combined clinics.

\section{Appendix 4. Haematopoietic SCT for $\mathrm{SCD}^{[14,15]}$}

Allogeneic SCT offers the only chance of cure in SCD. Gene therapy is still in the development phase and is currently not a realistic option. The indications for transplantation in SCD remain poorly defined owing to the variable phenotypic expression of the disease with age. The general experience in transplantation for this disease is also less than in thalassaemia. As a rule, in the absence of severe organ dysfunction, this treatment option should be considered in all moderately or severely symptomatic children if an HLA-identical donor is available. Results are better if transplantation is performed at an earlier age, particularly before irreversible sickle vasculopathy is established.

While the outcome of transplantation for SCD has been favourable, the main limiting factors for its wider use are lack of HLAcompatible donors, infrastructure and expertise, and cost. Outcomes depend on certain risk factors associated with the treatment of the haemoglobinopathy, such as irregular iron chelation therapy, which significantly increases transplant-related mortality. In addition, transplantation for SCD may have unique complications such as cerebrovascular occlusive/haemorrhagic episodes with stroke, convulsions requiring prophylaxis with anticonvulsive therapy, meticulous control of electrolytes, and maintaining higher than baseline blood haemoglobin levels and platelet counts throughout the procedure.

For the success of this type of therapy, two barriers must be overcome, viz. graft rejection and graft-versus-host disease (GVHD). For effective engraftment myeloablative conditioning is preferred, particularly in view of the highly cellular marrow as a consequence of chronic haemolysis and the brisk immune response resulting from exposure to allogeneic antigens during blood transfusions. The specifics of myeloablation are beyond the scope of this review.

The probabilities of overall survival, sickle cell-free survival and transplant-related mortality are $92 \%, 86 \%$ and $5 \%$, respectively. Despite the appropriate use of immunosuppressive agents, approximately one-third of patients will develop clinically relevant scores of GVHD, necessitating further therapy with corticosteroids and immunosuppressive agents. Adult patients usually have more advanced disease, and therefore greater comorbidities and higher treatment-related mortality.

A proportion of patients may have re-emergence of their own haematopoiesis with persistent and stable chimerism, which is generally associated with an asymptomatic state. In contrast to transplantation for malignancies, mixed chimerism in haemoglobinopathies therefore does not necessarily mean treatment failure. Allogeneic SCT is an effective treatment option, and currently the only proven curative therapy for patients with SCD. By removing the sickle cell phenotype, the vasculopathy is reversed and patients are likely to have a normal quality of life. Challenges remain the broader availability of this procedure with the use of alternative donors, as well as enhancing engraftment and preventing GVHD. 